\title{
Virtual topology design issues for variable traffic
}

\author{
T. Wauters, ${ }^{\text {a) }}$ D. Colle, E. Van Breusegem, S. Verbrugge, \\ S. De Maesschalck, J. Cheyns, M. Pickavet, and P. Demeester \\ Dept. of Information Technology (INTEC), Ghent University - IMEC, \\ Sint-Pietersnieuwstraat 41, 9000 Ghent, Belgium
}

a)tim.wauters@intec.ugent.be

\begin{abstract}
This paper discusses and evaluates, in terms of number of wavelength channels and router port count, different grooming strategies exploiting the benefits of statistical multiplexing. For the network design, a hybrid solution, combining the advantages of both the end-to-end and the link-by-link grooming scenario, is proposed.
\end{abstract}

Keywords: traffic grooming, variable traffic

Classification: Science and engineering for electronics

\section{References}

[1] R. Guérin, H. Ahmadi, and M. Naghshineh, "Equivalent Capacity and Its Application to Bandwidth Allocation in High-Speed Networks," IEEE J. Select. Areas Commun., vol. 9, no. 7, Sept. 1991.

[2] E. Van Breusegem et al, "Overspill Routing In Optical Networks: a new architecture for future-proof IP over WDM networks," Fourth Annual Optical Networking and Communications Conference, Dallas (USA), Oct. 2003.

\section{Introduction}

When designing a virtual topology, a key issue is to groom the traffic in the logical links in such a way that a good compromise between capacity efficiency and node cost is achieved. Two extreme grooming strategies exist. In endto-end grooming, a dedicated logical link is used for each traffic demand, possibly resulting in a full-mesh virtual topology. In link-by-link grooming, each network node terminates all logical links entering that node: the virtual topology corresponds to the physical topology.

Variable traffic can be modelled using distributions. For example, the demands from one of the left nodes to the right node in Figure 1 requires on average the capacity of 2.5 circuits, but per demand only $2.5 C-A$ of the capacity is (almost) always needed, while up to $2.5 C+A$ of the capacity is sporadically required. Grooming or aggregating variable traffic leads to a smaller variability relative to the average value: indeed, the variance typically follows a square-root dependency while the average traffic volume grows linearly [1]. 
A good approximation for the aggregated capacity needed to transport $N$ traffic flows with an average of $m_{i}$ and a standard deviation of $\sigma_{i}$, so that no more than a fraction $\varepsilon$ of the traffic gets lost, is given by:

$$
C_{a}=M+\alpha \cdot \sigma \quad \text { with } \quad \alpha=\sqrt{-2 \ln (\varepsilon)+\ln (2 \pi)} .
$$

In this equation $M$ is the mean aggregate traffic rate and $\sigma$ is the standard deviation of the aggregate traffic:

$$
M=\sum_{i=1}^{N} m_{i} \quad \text { and } \quad \sigma^{2}=\sum_{i=1}^{N} \sigma_{i}^{2} .
$$

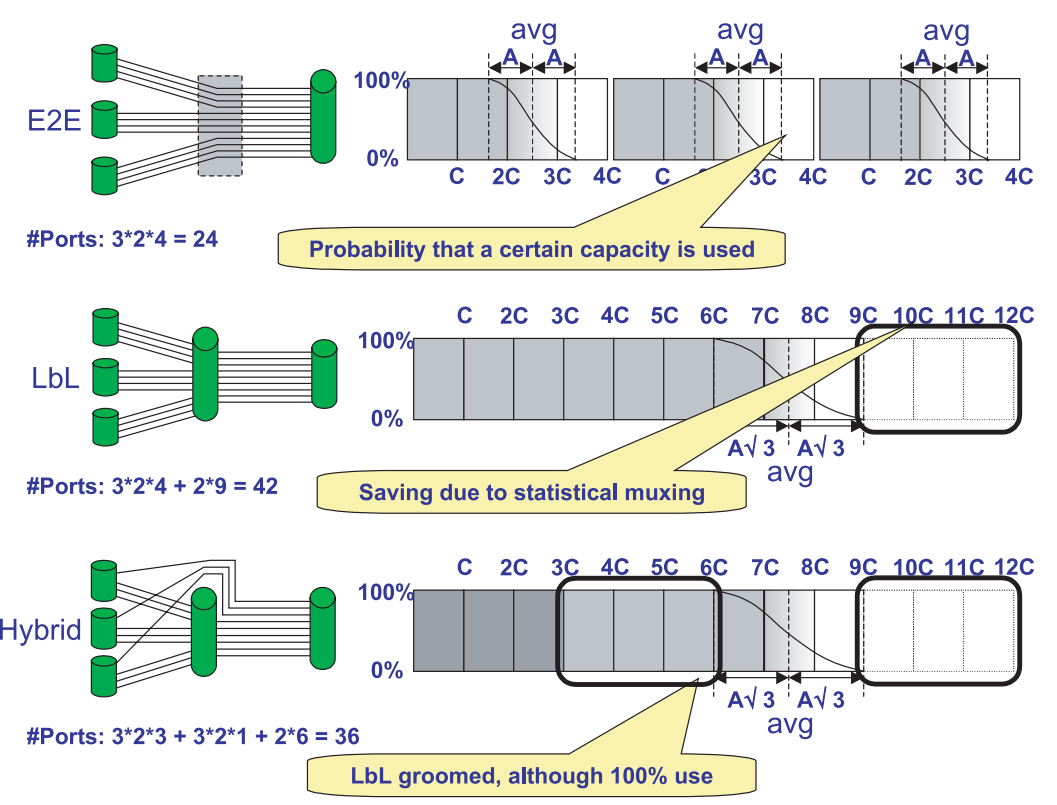

Fig. 1. End-to-end versus link-by-link grooming. The hybrid scenario combines the advantages of both strategies $(C$ : capacity of a circuit/lightpath).

The example of Figure 1 (top part) illustrates how this feature can be exploited in order to decrease capacity/investment costs. With end-to-end grooming, each of the three demands needs 4 wavelength channels that are all cross-connected in the middle node: $3 * 4=12$ wavelength channels are needed on the link between the middle and right node. However, in the linkby-link grooming case, the three demands are aggregated in the same logical link between the middle and right node, only requiring $2.5 C * 3+A * \sqrt{3}=$ $7.5 C+A * \sqrt{3}$ channels. Thus, when $A<=1.5 / \sqrt{3}$, only 9 instead of 12 wavelength channels are needed on the fibre between the middle and right node.

Figure 1 also shows that, although link-by-link grooming benefits from the statistical multiplexing gain, it requires more router ports (expensive O/E interfaces): 42 instead of 24 router ports (thus an increase of almost $100 \%)$. This very simple design strategy did however not take into account that each end-to-end demand always needs more than one circuit/wavelength 
channel. And thus (see Figure 1, bottom part), there is no need to terminate these completely filled lightpaths (dark grey capacity) in the middle node and to process each individual packet carried in these lightpaths. Thus, by cross-connecting lightpaths filled up for $100 \%$, up to $2 * 3=6$ router ports can be saved in the middle node, without impacting the statistical multiplexing gain.

At the bottom of Figure 1 we consider only lightpaths with a filling of exactly $100 \%$ to be cross-connected in the middle node (thus a single wavelength channel per demand). However cross-connecting a second wavelength channel/circuit per demand would probably also make sense, since these circuits are also nearly completely filled. Of course, this would probably require an additional wavelength channel on the link between the middle and right node (and thus two additional router ports), but cross-connecting these three wavelength channels in the middle node will save there $2 * 3=6$ router ports. Thus, this would result in a net gain of $6-2=4$ router ports.

It might be interesting to reuse the unused capacity of these crossconnected lightpaths to transport some other packets (routed hop-by-hop) by marking these packets with an orthogonal label (e.g., FSK label): this is called Overspill Routing In Optical Networks (ORION) [2].

\section{Evaluation}

To evaluate the different options discussed in the previous section, we consider a tree network, consisting of 8 levels. A node in level $N$ connects to 5 nodes in level $N-1$. It is assumed that between each leaf node and the root node on average a set of 10 streams have to be routed and that the capacity of a wavelength channel is equivalent to the bandwidth of 100 streams.

\subsection{Hybrid grooming scenario}

Figure 2 shows the total number of router ports needed for different levels of traffic variability. The figure compares pure end-to-end grooming, pure linkby-link grooming and a hybrid end-to-end/link-by-link grooming design. The latter situation corresponds to what has been presented at the bottom of in Figure 1: wavelength channels that are completely filled at level $N$ are crossconnected in all higher levels. Thus in Figure 1, level 2 would cross-connect only 3 wavelength channels, while level 3 would cross-connect 6 wavelength channels, due to the statistical multiplexing gain at level 2. In the peak design it is assumed that no statistical multiplexing gain can be achieved (the variance proportionally depends on the average traffic volume), while in the statistical design a square-root dependency for the variance results in the obtained statistical multiplexing gain.

As the figure shows, the larger the traffic variability relatively to the average traffic volume, the larger this statistical multiplexing gain (in this example up to $23 \%$ and $42 \%$ in respectively the link-by-link and hybrid grooming designs). Of course, in case of no variability, there exists no difference between the peak and statistical design. Note also that the hybrid grooming 
design is closest (but not equal due to the granularity mismatch between the demands and the wavelength channel capacity) to the end-to-end grooming design in case of no traffic variability and moves more and more to the link-by-link grooming design as the traffic variability grows.

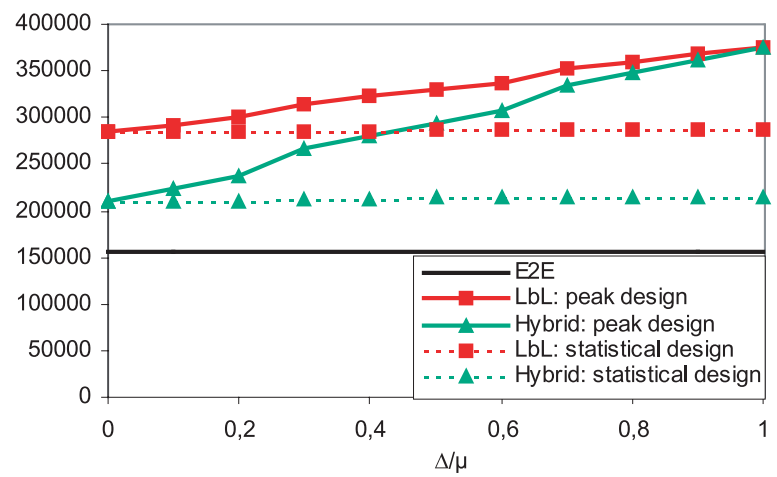

Fig. 2. Number of router ports for increasing traffic variability. The link-by-link and the hybrid strategies are compared to the end-to-end scenario, for peak and statistical design.

The hybrid and link-by-link grooming designs would become identical when at the pen-ultimate level, not a single wavelength channel gets crossconnected. This would be the case for a much higher traffic variability in the statistical design compared to the $100 \%$ variability in the peak design (i.e., proportional dependency between average and variance).

\subsection{Network design}

Figure 3 considers the same network scenario as in Figure 2 and assumes a relative traffic variability of $20 \%$. As explained at the end of section 1 , it can be interesting to also cross-connect highly (but not only completely filled) wavelength channels in the hybrid design. For this purpose we split the network in two parts: the first $N$ levels are designed according to the hybrid grooming strategy (cross-connection of only completely filled channels) and the remaining $8-N$ levels are designed according to the end-to-end grooming strategy. It is clear from the figure that up to level 3 the statistical multiplexing gain is dominated by the impact of the grooming due to the granularity mismatch between the demands and the wavelength channel capacity. A depth up to level 4 realizes a statistical multiplexing gain of $13 \%$ (compared to the peak design) in terms of wavelength channels entering the root node, at the price of less than $2 \%$ more router ports compared to the design with a depth up to level 3.

Extending the hybrid design even further will never lead to a statistical multiplexing gain higher than $17 \%$, requiring $1 \%$ more router ports compared to a depth up to level 4 . 


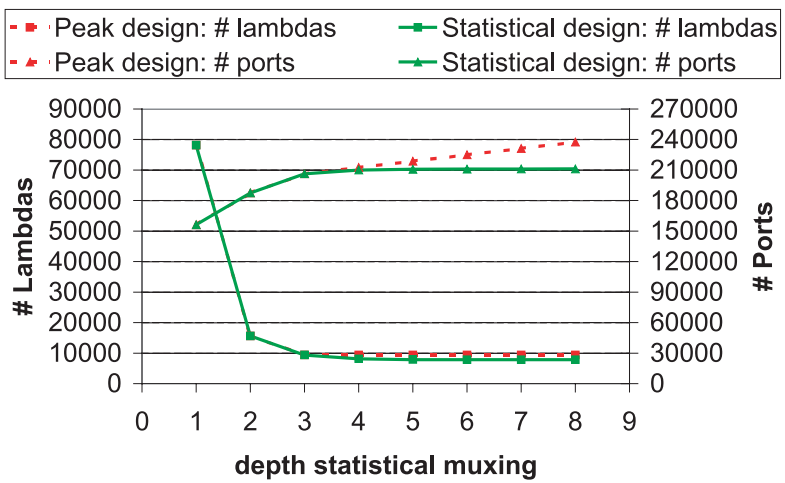

Fig. 3. Increasing number of levels $(N)$ assuming the hybrid grooming strategy, allowing for statistical multiplexing gain.

\section{Conclusions}

We have demonstrated that an important saving in wavelength channels and router ports can be achieved due to the statistical multiplexing gain in case of variable traffic, by comparing the peak and the statistical design. The hybrid end-to-end/link-by-link grooming strategy was found to combine the benefits of statistical multiplexing of the link-by-link grooming strategy and the lower number of router ports of the end-to-end grooming design.

By cross-connecting highly and not only completely filled wavelength channels in this hybrid scenario, a dominant fraction of the highest possible statistical multiplexing gain can already be realized at the price of a relative small router port penalty.

\section{Acknowledgments}

This work was partly funded by the EC through the IST-projects NOBEL, ePhoton/ONe and STOLAS, by the Flemish government through the FWO project G.0315.04, the IWT-project ONNA, the IWT/ITEA-project TBONES and by Ghent University through the BOF-project RODEO.

D. Colle, E. Van Breusegem and S. Verbrugge thank the IWT for its financial support through their post-doctoral/PhD grants. J. Cheyns is a Research Assistant with the Flemish Fund for Scientific Research. 